\title{
Europa in der Krise? Vorwort zum Sonderheft 5 der Zeitschrift für Vergleichende Politikwissenschaft
}

\author{
Markus Steinbrecher · Evelyn Bytzek • Ulrich Rosar · Sigrid Roßteutscher
}

Online publiziert: 20. September 2014

(C) Springer Fachmedien Wiesbaden 2014

Niedrige Beteiligungsraten bei Europawahlen, Stimmengewinne für europaskeptische Parteien bei Wahlen auf nationaler und europäischer Ebene, das Aufbrechen längst vergessen geglaubter Vorurteile und Konflikte zwischen europäischen Partnern, inkonsistente Bevölkerungseinstellungen zu den Euro-Rettungsmaßnahmen und wachsende Skepsis gegenüber der Europäischen Zentralbank und anderen europäischen Institutionen sind nur einige der Symptome der als „Eurokrise“ oder „Staatsschuldenkrise" bezeichneten Krise der letzten Jahre in Europa, die sich auch bei der

\footnotetext{
Dr. M. Steinbrecher $(\square)$

Fakultät für Sozialwissenschaften, Lehrstuhl für Vergleichende Politische Verhaltensforschung, Universität Mannheim,

A 5,6,

68131 Mannheim, Deutschland

E-Mail: markus.steinbrecher@uni-mannheim.de

Dr. E. Bytzek

Institut für Sozialwissenschaften, Abteilung Politikwissenschaft, Universität Koblenz-Landau, Kaufhausgasse 9,

76829 Landau in der Pfalz, Deutschland

E-Mail: bytzek@uni-landau.de

Prof. Dr. U. Rosar

Philosphische Fakultät, Institut für Sozialwissenschaften Abteilung Soziologie, Universität

Düsseldorf,

Universitätsstr. 1, Gebäude 23.31,

40225 Düsseldorf, Deutschland

E-Mail: ulrich.rosar@uni-duesseldorf.de

Prof. Dr. S. Roßteutscher

Fachbereich Gesellschaftswissenschaften, Institut für Soziologie und Institut für

Politikwissenschaft, Schwerpunkt soziale Konflikte und sozialer Wandel,

Goethe-Universität Frankfurt am Main,

Grüneburgplatz 1- Hauspostfach 15,

60323 Frankfurt am Main, Deutschland

E-Mail: rossteutscher@soz.uni-frankfurt.de
} 
Europawahl 2014 deutlich gezeigt haben. Die Geschwindigkeit der Krisengipfel, die Komplexität der ökonomischen und politischen Zusammenhänge sowie die teilweise zweifelhafte rechtsstaatliche und demokratische Legitimation der Rettungsmaßnahmen machen es nicht nur der Politik und den Bürgerinnen und Bürgern schwer, den Ereignissen zu folgen und sie zu verstehen. Daraus ergeben sich auch zahlreiche Herausforderungen für die vergleichende Wahl- und Einstellungsforschung.

So stellt sich beispielsweise die Frage, ob sich die durch Massendemonstrationen in vielen europäischen Ländern und in den Ergebnissen verschiedener Umfragen sichtbar werdende, wachsende euroskeptische Stimmung in dauerhaften Veränderungen des Niveaus politischer Unterstützung für die europäische Integration und die Institutionen der EU niederschlägt. Damit verbunden ist ein großes Forschungsinteresse daran, ob hergebrachte Erklärungsmuster und theoretische Modelle wie der utilitaristische Ansatz, kognitive Mobilisierung sowie der Politisierungsansatz (Gabel 1998; Gabel und Palmer 1995) die Unzufriedenheit, die zeitliche Entwicklung politischer Unterstützung und länderspezifische Unterschiede im Niveau europafreundlicher und -skeptischer Einstellungen (immer noch) erklären können.

Politische Systeme sind generell auf ein Mindestmaß an Unterstützung durch ihre Bürgerinnen und Bürger angewiesen (Almond und Verba 1965; Easton 1965, 1975). Für ein immer noch relativ junges politisches System wie das der EU ist die politische Befürwortung durch seine Bürgerinnen und Bürger von größerer Bedeutung als für andere politischen Ebenen oder Einheiten. Gründe dafür sind das Fehlen klassischer Eigenschaften politischer Staatlichkeit (McCormick 2008), die geringere wahrgenommene Wichtigkeit der europäischen im Vergleich zur nationalen oder subnationalen Ebene (Decker et al. 2013, S. 42) sowie das immer noch existierende demokratische Defizit der EU. Hinzu kommt, dass die europäische Einigung weitgehend ein Projekt der politischen Eliten geblieben ist. Das Verblassen des bis in die 1990er Jahre vorherrschenden permissiven Konsenses zwischen Eliten und Bevölkerung (Lindberg und Scheingold 1970) zeigt sich immer wieder in integrationshemmenden Entscheidungen der Bürgerinnen und Bürger in Referenden oder Wahlen.

Dieses Sonderheft untersucht daher Positionen, Meinungen, Einstellungen und politisches Verhalten der Bürgerinnen und Bürger im Kontext der EU und der europäischen Integration aus verschiedenen Perspektiven. Besonders im Mittelpunkt stehen dabei in vielen Beiträgen die möglichen Folgen der Eurokrise. Die Mehrheit der hier versammelten Aufsätze ist im Kontext der Jahrestagung des Arbeitskreises „Wahlen und politische Einstellungen“ der Deutschen Vereinigung für Politische Wissenschaft (DVPW) in Mannheim im Juni 2013 entstanden. Zusätzlich wurden weitere Beiträge über eine offene Ausschreibung eingeworben.

Im ersten Teil des Sonderheftes konzentrieren sich die Autorinnen und Autoren auf die Erklärung der Unterstützung für die europäische Integration und Institutionen der EU im Kontext der Krise. So können Nicola Bücker und Tuuli-Marja Kleiner zeigen, dass Vertrauen für die Institutionen der EU sowohl auf der Basis der Leistungen europäischer Institutionen und des wahrgenommenen Nutzens der europäischen Integration entsteht, gleichzeitig aber auch eine Übertragung von Vertrauen vom nationalen politischen System auf die europäische Ebene stattfindet. Toni Schell untersucht, ob sich ökonomische Einflussfaktoren während der Eurokrise stärker auf instrumentelle Einschätzungen und Einstellungen zur Vertiefung der europäischen Integration 
auswirken. Seine Ergebnisse zeigen, dass die europäischen Bürger nicht in besonderer Weise auf die Krise zu reagieren scheinen, denn ökonomische Indikatoren wie Veränderungen der Arbeitslosenrate oder des Bruttoinlandsprodukts werden während der Krise als Determinanten europaspezifischer Einstellungen nicht wichtiger. Auch Philipp Runge kann verdeutlichen, dass die Krise nicht zu einer systematischen Verschlechterung der Wahrnehmung der europäischen Integration durch die Bürger führt. Er konzentriert sich vor allem auf die Bedeutung von Identität für die Unterstützung der europäischen Integration und zeigt, dass europäische und nationale Identitäten zwischen 2006 und 2009 stärker werden, ihre Wirkung auf die Unterstützung der EU sich aber nur wenig verändert. Kai Theißen beschäftigt sich mit der Wahrnehmung der Krise durch die Bürgerinnen und Bürger in den Mitgliedsstaaten der EU. Wenig überraschend zeigen sich sehr unterschiedliche Bewertungen zwischen den von der Krise besonders betroffenen und den eher nicht betroffenen Ländern. Obwohl diese unterschiedlichen Bewertungen nicht zu einer kurzfristigen Abnahme der Unterstützung für die europäische Integration führen, sei langfristig aber nach Theißen mit einer möglichen Schwächung der Legitimationsgrundlage der EU zu rechnen. Fabian Endres untersucht die Einstellungen der Deutschen zum Euro während der Krise. Dies ist besonders relevant, da die Deutschen der gemeinsamen Währung von Beginn an skeptisch gegenüberstanden und ihnen bei den Rettungsmaßnahmen für die Krisenstaaten eine besondere Rolle zukommt. Endres' Ergebnisse zeigen, dass die Krise bisher nicht zu einer massiven Verschlechterung der Bewertung des Euro in Deutschland geführt hat. Er kann aber deutlich machen, dass insbesondere der weitere Verlauf der wirtschaftlichen Entwicklung in Deutschland und Europa sowie die Signale und das Handeln der politischen Eliten von großer Relevanz für die Einschätzungen der gemeinsamen Währung in Deutschland sind.

Der Beitrag von Endres bildet die Schnittstelle zum zweiten Teil des Sonderhefts, in dem es besonders um das Verhältnis zwischen Eliten und Bürgern geht. Aiko Wagner und Heiko Giebler untersuchen, ob eher die EU oder die nationale Regierung für die allgemeine wirtschaftliche Lage verantwortlich gemacht wird. Sie arbeiten dabei die besondere Bedeutung von Parteipräferenzen und EU-spezifischen Einstellungen heraus und können zeigen, dass gerade bei schlechter wirtschaftlicher Lage Anhänger der Regierungsparteien oder Menschen mit stark pro-europäischen Attitüden die jeweils andere politische Ebene in der Verantwortung sehen - ein demokratietheoretisch höchst relevanter Befund. Norma Osterberg-Kaufmann beschäftigt sich in ihrem Beitrag mit der Kongruenz der politischen Kultur zwischen Bürgern und Eliten. Ihr Beitrag ist sowohl aus inhaltlicher wie aus methodischer Sicht interessant: Sie nutzt Repertory Grids als innovative Methode zur Verschränkung qualitativer und quantitativer Forschungsansätze. Zudem kann sie zeigen, wie heterogen die Vorstellungen der Bürger zur Legitimität der EU und ihrer Institutionen sind.

Die Artikel im dritten Teil des Sonderhefts wechseln die Perspektive und konzentrieren sich auf die Erklärung politischen Verhaltens im europäischen Kontext. Reinhard Heinisch und Bernd Schlipphak analysieren die Effekte der Krise auf Euroskeptizismus und Wahlverhalten für euroskeptische Parteien in Ungarn und der Slowakei. Sie können für beide Länder eine leichte nationalistische Gegenreaktion auf die Krise und die europäische Integration feststellen, die zu größerem Skeptizismus führt. Der Beitrag von Ulrich Rosar und Markus Klein zeigt erstmals für Europawah- 
len die Bedeutung physischer Attraktivität von Kandidatinnen und Kandidaten. Bei der Europawahl 2004 hatten gut aussehende Kandidatinnen und Kandidaten größere Chancen auf einen Einzug ins Europaparlament. Zuletzt kann Markus Steinbrecher die Bedeutung europaspezifischer Einstellungen für die Wahlbeteiligung bei Europawahlen zwischen 1979 und 2009 nachweisen. Euroskeptische Länder weisen eine niedrigere Wahlbeteiligung auf, genauso wie es für europaskeptische Bürger wahrscheinlicher ist, nicht an einer Europawahl teilzunehmen. Allerdings sind europabezogene Perzeptionen nur eine Determinante der Wahlbeteiligung unter vielen und können alleine nicht die niedrige Teilnahmebereitschaft bei Europawahlen erklären.

Insgesamt zeigen die Ergebnisse der Beiträge in diesem Sonderheft, dass die Krise das Denken und Verhalten der Bürger im europäischen Kontext beeinflusst, aber nicht tiefgreifend verändert hat und dass Anhänger der europäischen Idee sich keine großen Sorgen über Stabilität und Erfolg des Einigungsprojekts machen müssen. Es ist allerdings anzumerken, dass die hier zusammengestellten Aufsätze und ihre Ergebnisse nur eine Momentaufnahme sein können. Die meisten Beiträge beziehen sich auf Umfragen oder Daten vor 2012, bieten also eher Informationen zu Einstellungen, Wahrnehmungen und Erklärungsmustern zu Beginn der Krise und beziehen die Europawahl 2014 nicht mit ein. Da die jüngsten Ergebnisse der Eurobarometer deutlich geringere Zustimmung zur europäischen Integration und sinkendes Vertrauen in europäische Institutionen zeigen (Europäische Kommission 2013) und die Europawahl 2014 in vielen Ländern zu Stimmengewinnen für Europaskeptiker und -gegner geführt hat, ergibt sich die Notwendigkeit und zusätzliches Potential für weitergehende Forschung zu politischen Einstellungen und Verhalten im Kontext der EU und der europäischen Einigung.

\section{Literatur}

Almond, Gabriel A., und Sidney Verba. 1965 [1963]. The Civic culture - political attitudes and democracy in five nations. Boston: Sage.

Decker, Frank, Marcel Lewandowsky, und Marcel Solar. 2013. Demokratie ohne Wähler? Neue Herausforderungen der politischen Partizipation. Bonn: Dietz.

Easton, David. 1965. A systems analysis of political life. New York: Wiley.

Easton, David. 1975. A re-assessment of the concept of political support. British Journal of Political Science 5:435-457.

Europäische Kommission. 2013. Die öffentliche Meinung in der Europäischen Union. Bericht Standard Eurobarometer 80. Brüssel: Europäische Kommission.

Gabel, Matthew. 1998. Public support for European integration: An empirical test of five theories. The Journal of Politics 60:333-354.

Gabel, Matthew J., und Harvey D. Palmer. 1995. Understanding variation in public support for European integration. European Journal of Political Research 27:3-19.

Lindberg, Leon N., und Stuart A. Scheingold. 1970. Europe's would-be polity: Patterns of change in the European community. Englewood Cliffs: Prentice-Hall.

McCormick, John. 2008. Understanding the European union. A concise introduction. 4. Aufl. Houndsmills: Palgrave Macmillan. 